CONFORMAL GEOMETRY AND DYNAMICS

An Electronic Journal of the American Mathematical Society

Volume 11, Pages 90-100 (June 11, 2007)

S 1088-4173(07)00159-2

\title{
REGULARITY OF GROWTH AND THE CLASS $\mathcal{S}$
}

\author{
D. DRASIN
}

Abstract. Given $1 / 2 \leq \mu \leq \rho \leq \infty$, there is an entire function $f(z)$ in the Speiser class $\mathcal{S}$ of order $\rho$, lower order $\mu$. $f$ may have as few as three singular values.

\section{INTRODUCTION}

An entire (or meromorphic) function $f(z)$ belongs to the class $\mathcal{S}$ (for Speiser) if its singularities project onto the finite set $A=\left\{a_{1}, \ldots, a_{q}\right\}$. Thus if $a \notin A$, then whenever $f\left(z_{0}\right)=a$, there are neighborhoods $N_{1} \ni z_{0}$ and $N_{2} \ni a$ such that $f$ has a local inverse $f^{-1}: N_{2} \rightarrow N_{1}$, with $f^{-1}(a)=z_{0}$. The class $\mathcal{S}$ includes most of the familiar analytic/meromorphic functions, and has some remarkable properties, which place $\mathcal{S}$ between rational functions and general meromorphic functions. For example, if $f \in \mathcal{S}$, then the Fatou set of its iterates contains no wandering domain (4] for entire functions and [1] for meromorphic functions); in addition, if $f \in \mathcal{S}$, the inequality which forms the Nevanlinna second fundamental theorem becomes an asymptotic equality [7].

Our result here shows that this principle has some limitation. Recall that the order $\rho$ (lower order $\mu$ ) of an entire function is

$$
\rho(\mu)=\limsup _{r \rightarrow \infty}\left(\liminf _{r \rightarrow \infty}\right) \frac{\log \log M(r, f)}{\log r}=\limsup _{r \rightarrow \infty}\left(\liminf _{r \rightarrow \infty}\right) \frac{\log T(r)}{\log r} .
$$

Theorem 1. There exist entire functions in $\mathcal{S}$ of irregular growth: given $1 / 2 \leq$ $\mu<\rho \leq \infty$, there is a function $f \in \mathcal{S}$ of order $\rho$ and lower order $\mu$.

This question was raised by Adam Epstein. That necessarily $\mu \geq 1 / 2$ follows from 2].

Sergiy Merenkov [6] has shown that there are entire functions in $\mathcal{S}$ whose maximum modulus grows arbitrarily rapidly. Even when $\rho=\mu=\infty$, our function will have restricted growth, since $\left\|h^{\prime \prime}\right\|_{\infty} \leq 1 /(3 \pi)$; however, we are able to specify the behavior of the characteristic $T(r, f)$ (as well as the maximum modulus) rather precisely.

Standard notation (here we assume the variable is $z$, but the notation will also be used with other variables, the context making clear the appropriate choice): $B(r)=\{|z| \leq r\} ; S(r)=\{|z|=r\} ; B=B(1) ; S=S(1)$.

Received by the editors July 11, 2006.

This work was initiated in 2001 when at the University of Nottingham, under SRC support. Presented December 3, 2005 at the Quasifest (Helsinki) in honor of the anniversaries of Seppo Rickman and Jussi Väisälä. The author thanks both Mathematics Departments for their hospitality and atmosphere. 


\section{A STRIP MAPPING}

The construction depends on an explicit mapping (Proposition 1)

$$
\varphi: \Sigma \rightarrow T,
$$

where $\Sigma$ is the strip $\{(x, y) ; h(x)<y<h(x)+2 \pi\}$ and $T$ the standard strip, which we take as $\{(u, v) ;|v|<\pi / 2\}$. The function $h$ is smooth, $h^{\prime} \geq 0$, and $h(x) \equiv 0(x \leq 1)$.

With $\mu$ and $\rho$ as in the statement of the theorem, we take $h$ so that if

$$
M(x)=\frac{1}{2} \int_{0}^{x}\left(1+h^{\prime}(s)^{2}\right)^{1 / 2} d s
$$

then

$$
\mu=\liminf _{x \rightarrow \infty} \frac{M(x)}{x} \leq \limsup _{x \rightarrow \infty} \frac{M(x)}{x}=\rho .
$$

In turn, $h$ determines a strip $\Sigma$ bounded by $\operatorname{arcs} \Gamma, \Gamma^{\prime}: \Gamma=\{(x, h(x))\}, \Gamma^{\prime}=$ $\{(x, h(x)+2 \pi)\}, x \in \mathbb{R}$.

In $\S 6, h$ will be constructed in stages, over intervals $J_{n}=\left[x_{n}, x_{n+1}\right]_{n \geq 0}$, where $x_{0} \geq 1$ and the lengths of the $\left\{J_{n}\right\}$ may be taken as large as needed. The exposition is a bit simpler when $\rho<\infty$, but it is more direct to discuss the most general case. Thus choose a sequence $\rho_{n}^{*} \uparrow \rho, \rho_{n}^{*}<\infty$ for all $n$. We then require that

$$
\inf _{J_{n}} h^{\prime}(t)=\sqrt{2 \mu-1}+(1 / n), \sup _{J_{n}} h^{\prime}(t)=\sqrt{2 \rho_{n}^{*}-1},
$$

and note that (3) is compatible with the intervals $J_{n}$ being large, and $\eta_{n}$ in (4) small. We suppose à priori that $\left\|h^{\prime \prime}\right\|_{\infty} \leq 1 /(3 \pi)$, and then introduce a positive sequence $\left\{\eta_{n}\right\}$ so that

$$
\begin{gathered}
h^{\prime}(x)\left|h^{\prime \prime}(x)\right|<\eta_{n}, \quad\left|h^{\prime \prime}(x)\right|<\eta_{n} \quad\left(x \in J_{n}\right), \\
\left|h^{\prime \prime}(x)\right|<\eta_{n} h^{\prime}(x) \text { whenever } x \in J_{n} \text { and } h^{\prime}(x)>1 .
\end{gathered}
$$

Many estimates contain expressions in which $o(1)$ appears. The estimate

$$
A=o(1) B \quad(\eta)
$$

is shorthand for the statement that the error terms can be controlled for $x \in J_{n}$ by an expression which depends only on the $\left\{\eta_{n}\right\}$ in (4).

The strip $\Sigma$ admits a natural foliation. For each $x$ construct the perpendicular segment $\mathcal{L}=\mathcal{L}(x)$ to $\Gamma$ connecting boundary points $(x, h(x))$ and $(L(x), h(L(x))+$ $2 \pi) \in \Gamma^{\prime}$ through $\Sigma$, thus implicitly defining $L(x)$, and let $\ell(x)$ be the length of $\mathcal{L}(x)$. It is clear that $L$ is unique: were $u_{0}$ and $t_{0}$ suitable possibilities for $L\left(x_{0}\right)$, $u_{0} \neq x_{0}, t_{0} \neq x_{0}$ with $t_{0}<u_{0}$, then

$$
0<\frac{h\left(u_{0}\right)-h\left(t_{0}\right)}{u_{0}-t_{0}}=-\frac{1}{h^{\prime}\left(x_{0}\right)},
$$

a contradiction since $h$ is nondecreasing.

Lemma 1. $L$ is a smooth function of $x$ with

$$
0 \leq x-L(x) \leq 4 \pi,
$$

and

$$
L^{\prime}(x)=1+o(1) \quad(\eta)
$$


Remark. Recall that the qualification $(\eta)$ here and below means that the error terms depend only on the data (41).

Proof. We bound $x-L(x)$. Consider the triangle with vertices

$$
A=(x, h(x)), B=(x, h(x)+2 \pi), C=(L(x), h(L(x))+2 \pi),
$$

and let $D$ be the point in the segment $A B$ with $A B \perp C D$ (since $h^{\prime}>0$ we have that $\Im A<\Im C<\Im B)$.

First suppose that $h^{\prime}(x) \leq 2$. Then $A C$ has slope $<-1 / 2$, and so (the continuation of) this segment meets the horizontal line $\{\Im z=h(x)+2 \pi\}$ at a point $(p, h(x)+2 \pi)$, where $x-p \leq 4 \pi$. Thus, in this case $x-L(x)=|C D|<4 \pi$.

Otherwise, $h^{\prime}(x) \geq 2$, and since $\left\|h^{\prime \prime}\right\|_{\infty} \leq 1 /(3 \pi)$, we have that $h^{\prime}(t) \geq 1 / 2$ for $x-t<4 \pi$. This means that the horizontal line segment joining $(x, h(x))$ to $(x-4 \pi, h(x))$ passes through $\Gamma^{\prime}$, and so forces $x-L(x) \leq 4 \pi$.

Next, choose $x_{0}, t_{0}$ with $t_{0}=L\left(x_{0}\right)$. Consider for $x$ near $x_{0}$ the function

$$
F(x, t)=h^{\prime}(x)(h(t)-h(x)+2 \pi)-(x-t) .
$$

If $t_{0}=L\left(x_{0}\right)$, it follows that $F\left(x_{0}, t_{0}\right)=0$, and clearly

$$
\frac{\partial F}{\partial t}=h^{\prime}(x) h^{\prime}(t)+1 \geq 1
$$

Thus for $x$ near $x_{0}$, the equation $F(x, t)=0$ has a unique solution $t=t(x)$ which is continuous. The implicit function shows that $t(x)$ is smooth:

$$
\frac{d t}{d x}=L^{\prime}(x)=-\frac{F_{x}}{F_{t}}=\frac{1+h^{\prime}(x)^{2}+h^{\prime \prime}(x)(h(x)-h(t)-2 \pi)}{1+h^{\prime}(x) h^{\prime}(t)}
$$

and $L^{\prime}(x)=1+o(1)$, with the error terms as described.

Corollary 1. If $\ell(x)$ is the length of $\mathcal{L}(x)$, then

$$
\ell(x)=(1+o(1)) \frac{2 \pi}{\sqrt{1+h^{\prime}(x)^{2}}} \quad(\eta) .
$$

Proof. Let $\Delta$ be the triangle from Lemma 1, so that $\angle(C A B)=\alpha=\tan ^{-1} h^{\prime}(x)$. Moreover, the slope of $C B$ is $(h(x)-h(L(x))) /(x-L(x))=h^{\prime}(\xi)$ with $x-\xi=O(1)$, and so if $\beta=\angle(C B A)$, then $\beta=\pi / 2-\tan ^{-1} h^{\prime}(\xi)$ :

$$
\sin \beta=\frac{1}{\sqrt{1+h^{\prime}(\xi)^{2}}}=(1+o(1)) \frac{1}{\sqrt{1+h^{\prime}(x)^{2}}} .
$$


Now $\ell=|A C|$, and the (nearly right)-angle $\angle(A C B)$ opposite the vertical side of $\Delta$ is $\pi-(\alpha+\beta)$; thus the law of sines gives

$$
\begin{aligned}
\ell=\frac{2 \pi}{\sqrt{1+h^{\prime}(\xi)^{2}}} & \cdot \frac{\sqrt{1+h^{\prime}(x)^{2}} \sqrt{1+h^{\prime}(\xi)^{2}}}{1+h^{\prime}(x) h^{\prime}(\xi)} \\
& =(1+o(1)) \frac{2 \pi}{\sqrt{1+h^{\prime}(x)^{2}}}
\end{aligned}
$$

as claimed.

In addition to $L$, we use $L_{1}(x)$, defined so

$$
L\left(L_{1}(x)\right)=x
$$

thus the point $\left(L_{1}(x), h\left(L_{1}(x)\right)+2 \pi\right)=(x, h(x)+2 \pi)$ lies directly above $(x, h(x))$ on $\Gamma^{\prime} \subset \partial \Sigma$. The existence of $L_{1}$ follows from the discussion of $L$.

Lemma 2. The function $L_{1}$ satisfies

$$
L_{1}(x)-x=2 \pi(1+o(1)) \frac{h^{\prime}(x)}{1+h^{\prime}(x)^{2}} \quad(\eta) .
$$

Proof. Consider the triangle with vertices $A:(x, h(x)), B:\left(L_{1}(x), h\left(L_{1}(x)\right)\right)$, $C:(x, h(x)+2 \pi) \equiv\left(L\left(L_{1}(x)\right), h\left(L\left(L_{1}(x)\right)+2 \pi\right)\right)$, so that now

$$
\angle A C B=\tan ^{-1} h^{\prime}\left(L_{1}(x)\right) .
$$

Since $L_{1}(x)-x<4 \pi$, the corollary yields

$$
L_{1}(x)-x=\ell\left(L_{1}(x)\right) \sin \beta=(1+o(1)) \frac{2 \pi}{\sqrt{1+h^{\prime}\left(L_{1}(x)\right)^{2}}} \cdot \frac{h^{\prime}\left(L_{1}(x)\right)}{\sqrt{1+h^{\prime}\left(L_{1}(x)\right)^{2}}},
$$

and the conclusion follows from (44).

We next show

Proposition 1. The mapping

$$
\varphi: \mathcal{L}(x) \rightarrow\{\Re w=M(x),|\Im w|<\pi / 2\},
$$

with $\varphi(x, h(x))=(M(x),-\pi / 2)$ and $|d w| /|d z|$ constant on $\mathcal{L}(x)$, is quasiconformal with dilatation

$$
\mu_{\varphi}(z)=\phi_{\bar{z}} / \phi_{z}=o(1) \quad(z \rightarrow \infty, z \in \Sigma) \quad(\eta) .
$$

Remark. Our definition of $\varphi$ forces $|d w|=|d z|$ when $z \in \Gamma$, and so (11) and (2) yield that $\lim \sup e^{M(x)}=e^{\rho x}, \liminf e^{M(x)}=e^{\mu x}$.

Proof. Consider $\psi=\varphi^{-1}$ and take $w_{0}=u_{0}+i v_{0} \in T$. Then $z_{0}=\left(x_{0}, y_{0}\right)=$ $\psi\left(w_{0}\right) \in \mathcal{L}(x)$ where $x$ satisfies the vector equation

$$
z_{0}-(x, h(x))=\frac{v_{0}+\pi / 2}{\pi} \mathcal{L}(x) \quad\left(\left|v_{0}\right|<\pi / 2\right) .
$$

If $z^{\prime}=\psi\left(w_{0}+i \tau\right) \in \mathcal{L}(x)$, then $z^{\prime}-z_{0}=(\tau / \pi) \mathcal{L}(x)$ and so

$$
\frac{\partial z}{\partial v}=\frac{\mathcal{L}(x)}{\pi}
$$


Next, with $w_{0}$ and $\tau$ as above, we have that if $\psi\left(w_{0}+\tau\right) \in \mathcal{L}\left(x^{\prime}\right)$, then

$$
\psi\left(w_{0}+\tau\right)-\psi\left(w_{0}\right)=\frac{v_{0}+\pi / 2}{\pi}\left(\mathcal{L}\left(x^{\prime}\right)-\mathcal{L}(x)\right),
$$

so (11) and Lemma 1 give that

$$
\frac{\partial z}{\partial u}=\frac{2+o(1)}{1+h^{\prime}(x)^{2}}\left(1, h^{\prime}(x)\right)
$$

and of course $\left(1, h^{\prime}(x)\right)$ is perpendicular to $\gamma$ at $(x, h(x))$. We thus deduce, using (5), that $\left|\psi_{u}\right|^{2}=\left(4 /\left(1+h^{\prime}(x)^{2}\right)\right)=(1+o(1))\left|\psi_{v}\right|^{2}$ :

$$
\left|\psi_{u}-i \psi_{v}\right|=o(1)\left|\psi_{u}\right| \quad(\eta)
$$

which yields the proposition.

A minor modification will be made to $\varphi$. Let $\varepsilon(x)$ (to be specified in (17)) be a positive smooth decreasing function such that $\varepsilon(x)=\varepsilon_{0}=\|\varepsilon\|_{\infty}<\pi / 4(x \leq 0)$ and

$$
\varepsilon(x) \rightarrow 0, \quad \varepsilon^{\prime}(x) \rightarrow 0 \quad(x \rightarrow+\infty) .
$$

On recalling (3) we suppose in addition for $x \in J_{n}$ that

$$
\varepsilon\left(x_{n}\right) \sqrt{2 \rho_{n}^{*}-1}<1 \quad(n \geq 0) ; \quad \varepsilon\left(x_{n}\right) \sqrt{2 \rho_{n}^{*}-1} \rightarrow 0 \quad(n \rightarrow \infty) .
$$

Now consider the (modified) strip

$$
\Sigma^{*}=\{(x, y) ; h(x)+\varepsilon(x) \leq v \leq h(x)+2 \pi\} .
$$

For sufficiently small $\varepsilon_{0}$ the mapping $p^{-1}: \Sigma \rightarrow \Sigma^{*}$ :

$$
p^{-1}(x, y)=\left(x, \frac{2 \pi-\varepsilon}{2 \pi} y+\varepsilon\left(1+\frac{h(x)}{2 \pi}\right)\right)
$$

is a $q c$ homeomorphism with dilatation

$$
\left|\mu_{p^{-1}}(z)\right|=O\left(\varepsilon(x)\left(1+h^{\prime}(x)\right)\right) \quad(z \in \Sigma) .
$$

This will be exploited in $\S 5$.

\section{The WINDING}

The strip $\Sigma^{*}$ corresponds to a spiraling region in the $Z=\exp z$-plane. Thus $\exp \Sigma^{*}$ is a connected set $\Sigma_{Z}$ whose intersection with each circle $S(R)(R>0)$ is an arc of angular measure $2 \pi-\varepsilon(\log R)$. We first study the composite map

$$
\Phi \equiv \exp \circ \varphi \circ p \circ \log : \Sigma_{Z} \rightarrow\{\Re W=U \geq 0\},
$$

which maps $\partial \Sigma_{Z}$ onto the imaginary $W=U+i V$ axis, normalized by $\Phi(0)=0$.

The explicit form of $\varphi$ shows that the $\Phi$-image of $S(R) \cap \Sigma_{Z}$ is an asymptotic semi-circle contained in $\{U \geq 0\}$ with endpoints at the points

$$
\left(0, e^{M\left(L_{1}(\log R)\right)}\right),\left(0,-e^{M(\log R)}\right) .
$$

Let $\partial^{-} \Sigma_{Z}, \partial^{+} \Sigma_{Z}$ be the arcs of $\partial \Sigma_{Z} \backslash\{0\}$, chosen such that for each $R>0$, $S(R) \cap \partial^{+} \Sigma_{Z}$ has the larger argument (measured through $\Sigma_{Z}$, this is consistent with the earlier notation $\partial^{ \pm} T$ ). Let $\tau^{-}, \tau^{+}$be arc-length on $\partial^{-} T, \partial^{+} T$, measured from 0 , and recall that $\Gamma$ is mapped to $\partial^{-} T$ under $\varphi$. Thus (1), (10) and the fact that each line $\{x=$ const. $\}$ is invariant under $p$ show that

$$
\frac{d(-V)}{d \log R}=e^{M(x)} \frac{1}{2}\left(1+h^{\prime}(x)^{2}\right)^{1 / 2} \quad(x=\log R) .
$$


When doing this computation on $\partial^{+} \Sigma_{Z}$, note that the point on $\partial^{+} \Sigma_{Z} \cap S(R)$ corresponds on $\partial^{+} T$ to $\left(L_{1}(x), h\left(L_{1}(x)\right)+2 \pi\right), x=\log R$. The computation just made, now with the estimates of $L^{\prime}$ from Lemma 1 and $L_{1}(x)-x$ from Lemma 2 now yield that $(x=\log R)$,

$$
\begin{gathered}
\quad \frac{d V}{d \log R}=\frac{d}{d x}\left(e^{M\left(L_{1}(x)\right)}\right)=e^{M\left(L_{1}(x)\right)} \frac{1}{2}\left(1+h^{\prime}\left(L_{1}(x)^{2}\right)\right)^{1 / 2}(1+o(1)) \\
=(1+o(1)) \frac{d(\log -V)}{d \log R} e^{(1 / 2) \int_{M(x)}^{M\left(L_{1}(x)\right)}\left(1+h^{\prime}(t)^{2}\right)^{1 / 2} d t} \quad\left(z \in \partial^{+} \Sigma_{Z}\right) \quad(\eta) .
\end{gathered}
$$

Now

$$
H(Z)=\exp \Phi(Z)
$$

maps $\Sigma_{Z}$ onto an unramified cover of $\{|W|>1\}$ with the only singularity of the inverse function being a single logarithmic branch point over $W=\infty$. Our normalization has $H(0)=1$.

We compute $n(R, i)$, the number of solutions to the equation $H(Z)=i$ with $Z \in \Sigma_{Z}$ (these points lie on the boundary of $\Sigma_{Z}$, but the counting function is well defined since, for example, the reflection principle may be applied on $\partial \Sigma_{Z}$ ). Thus if $n^{-}(R, i)$ and $n^{+}(R, i)$ are the number of such points in $B(R)$ whose $\Phi$-image is congruent to $0(\bmod 2 \pi i)$, then (11) and (12) show in turn

$$
n^{-}(R, i)=\frac{1}{2 \pi} e^{M(\log R)},
$$

and, more significantly,

$$
\begin{aligned}
n^{+}(r, i) & =e^{M\left(L_{1}(\log R)\right)} \\
& =(1+o(1)) \exp \left[(1 / 2) \int_{\log R}^{L_{1}(\log R)}\left(1+h^{\prime}(t)^{2}\right)^{1 / 2} d t\right] n^{-}(r, i),
\end{aligned}
$$

reflecting the more rapid covering of $S$ from $\partial^{+} \Sigma_{Z}$, due to the asymmetry of $\Sigma$, an effect frequently exploited (for example, see [5]). Lemma 2, (41) and the form of (11) show that the imbalance of coverings in (14) is controlled by

$\exp \left((1 / 2) \int_{\log R}^{L_{1}(\log R)}\left(1+h^{\prime}(s)^{2}\right)^{1 / 2} d s\right) \sim \exp \left((1+o(1)) \pi h^{\prime}(x)\left(1+h^{\prime}(x)^{2}\right)^{-1 / 2}\right) \quad(\eta)$.

In addition, if $|a|=1$, we check that $n(R, a)=n^{+}(R, i)+n^{-}(R, i)+O(1)$, the $O(1)$ uniform in $a$. Following the standard Nevanlinna theory, define $N(R, a)$ as

$$
(d / d \log r) N(r, a)=n(r, a),
$$

and (at least if $a \neq 1=H(0)) N(0, a)=0$.

A comparison of this with our controlling property (1) together with the remark following the statement of Proposition 1 at once give the next

Lemma 3. Let the characteristic of $H$ formally be defined as

$$
T(r, H)=\frac{1}{2 \pi} \int_{0}^{2 \pi} N\left(r, e^{i \phi}\right) d \phi
$$

(when $H$ is meromorphic, this is $H$. Cartan's formula). Then $H$ has order $\rho$ and lower order $\mu$. 
In the next section we extend $H$ to be quasiregular in the $z$ plane with singularities over three values, and satisfy the functional equation

$$
H(z)=f\left(\lambda^{-1} \zeta\right)
$$

where $f$ is entire and $\lambda$ is a $K$-quasiconformal homeomorphism of the plane. In this situation, we will find that Lemma 3 transfers at once to $f$.

\section{Extending $H$}

We have already noted that the spiraling of $S_{Z}$ in the $Z$-plane is reflected in $H$ covering $S$ faster on $\partial^{+} S_{Z}$, but now exploit (3) to arrange that this holds on the infinitesimal level.

Lemma 4. For $n \geq 1$ we may choose $\eta_{n}>0$ in (4), but sufficiently small to ensure that

$$
\frac{d n^{+}(t)}{d t}>\frac{d n^{-}(t)}{d t} \quad(t>1)
$$

Proof. According to the computations leading to (12), we have

$$
\frac{d n^{+}(t)}{d t}=(1+o(1)) e^{M\left(L_{1}(t)\right)-M(t)} \frac{d n^{-}(t)}{d t} \quad(\eta)
$$

and on recalling (3), Lemma 2, we have

$$
M\left(L_{1}(t)\right)-M(t)=\pi(1+o(1)) h^{\prime}(t)\left(1+h^{\prime}(t)^{2}\right)^{-1 / 2} \quad(\eta) .
$$

However, when $t \in J_{n}$, (3) asserts that $h^{\prime}(t)$ has absolute positive upper and lower bounds, while all expressions $o(1)$ are controlled by $\eta_{n}$, which until this moment has not been assigned. We now do this to guarantee that the lemma holds.

For $k \geq 1$ we mark as $Z_{k}^{-}, Z_{k}^{+}$the points on $\partial \Sigma_{Z}$ which correspond to $W= \pm i$ under $H$ as $|Z|$ increases. This is done with $Z_{k}^{ \pm} \in \partial^{ \pm} \Sigma_{Z}$ and $H\left(Z_{1}^{ \pm}\right)= \pm i$. In addition, set $Z_{0}=Z_{0}^{+}=Z_{0}^{-}=0$.

This induces a partition of the $Z$-plane into concentric annuli $A_{k}=\left\{R_{k} \leq|Z| \leq\right.$ $\left.R_{k+1}\right\}$ so that for $k \geq 1$, the $H$-image of $A_{k} \cap \partial^{-} \Sigma_{Z}$ covers $S^{+}:=S(1) \cap\{\Re W \geq 0\}$ or $S^{-}:=S \cap\{\Re W<0\}$ once. Thus, it follows from Lemma 4 that each interval $I_{k}^{+}$of $A_{k} \cap \partial^{-} \Sigma_{Z}$ may be matched to an interval $I_{k}^{+}$of $\partial^{+} \Sigma_{Z}$, having endpoints $Z_{k^{*}}^{+}, Z_{(k+1)^{*}}^{+} \subset \bigcup_{p} Z_{k}^{+}$with the properties:

(1) the $\left\{I_{k}^{+}\right\}$partition $\partial^{+} \Sigma_{Z}$,

(2) the $H$-image of each $I_{k}^{+}$covers $S^{+} \cup S^{-} q=q(k)$ times, and if $I_{k} \cap$ $\{|z|=\log x\} \neq \varnothing$ for some $x \in J_{n}$, then $1<q(k) \leq Q(n)<\infty$ (this requires both upper and lower bounds from (3) for $h^{\prime}(t)$ for $\left.t \in J_{n}\right)$,

(3) $H\left(Z_{k}^{-}\right)=(-1)^{k} i$,

(4) if $\Pi_{1}$ is the projection onto the $|Z|$-coordinate within $\Sigma_{Z}$, then

$$
\Pi_{1}\left(I_{k}^{-}\right) \cap \Pi_{1}\left(I_{k}^{+}\right) \neq \varnothing,
$$

(5) $H\left(Z_{k+1}^{+}\right)=-H\left(Z_{k}^{+}\right)$at the endpoints of each $I_{k}^{-}$and $H$ maps $I_{k}^{-}$to a simple cover of $S^{+}$or $S^{-}$. 
Thus $\partial I_{k}^{-}=\left\{Z_{k}^{-}, Z_{k+1}^{-}\right\}$, while only a subsequence of the $\left\{Z_{k}^{+}\right\}$are in $\bigcup_{k} \partial I_{k}^{+}$.

Note from (13) and the overriding condition (44) that (as usual, $x=e^{X}=e^{\Re Z}$ ), the image of

$$
\partial^{-} \Sigma \cap\left[x, x+(1+o(1)) \frac{2}{n(x)\left(1+h^{\prime}(x)^{2}\right)^{1 / 2}}\right]
$$

covers $S(1)$ once.

\section{Enter $\mathcal{S}$}

To extend $H$ to $\Sigma_{Z}^{\prime}$ and have irregular growth will force additional singularies of $H$ over at least two additional points, which, to have $H$ correspond to a function in $\mathcal{S}$, we take as $\pm p$, where $0<p<1$ is fixed ( $\operatorname{say} p=1 / 2$ ).

As a model, first consider a finite family of coverings of $B=B(1)$ in the $W$-plane in the range $2 \leq j \leq J\left(\rho^{*}\right)<\infty$ (recall (3) ), where we write $\rho^{*}$ in place of the more explicit $\rho_{n}^{*}$. This is based on $B_{j}$, the normalized covering of unit disk $B$ given by $W \rightarrow W^{j}$, which has one branch point (order $j-1$ ) over $W=0$. We view $\partial B_{j}$ as being composed of $2 j$ arcs, $j$ alternating over each of $S^{+}:=\{\Re W \geq 0\}$ and $S^{-}:=\{\Re W<0\}$ on a circuit of $\partial B_{j}$.

Although these $\left\{B_{j}\right\}$ would be the simplest class to use, they do not produce irregularity of growth. They are replaced by three related classes of quasiconformal images of the $B_{j}$, which are fused to extend $H$ to $\Sigma_{Z}^{\prime}$.

The first group is $B_{j}^{+}, B_{j}^{-}(j \geq 2)$, and we describe $B_{j}^{-}$; the only change for $B_{j}^{+}$ lies in the corresponding singular points being over $W=+p$. For each $j$ consider first the quasiconformal correspondence

$$
B_{j} \rightarrow B_{j}^{-}(0)
$$

which is the identity on the boundary and has the branch point $W=0$ shifted to $W=-p$. The dilatation of these maps can be taken to be bounded independent of $j$ (we have $W \rightarrow \Phi(W)$ with $\Phi$ qc on $S(1), \Phi(W)=W$ for $W \in S, \Phi(0)=-p$ ). Let $I$ be the (vertical) segment connecting $W= \pm i$, and choose two arcs of $\partial B_{j}$ over $S^{+}$which, on a circuit of $\partial B_{j}$, are separated by a single arc $S^{-}$(there are $j$ ways to do this). Then $B_{j}^{-}$is $B_{j}^{+}(0)$ with these two arcs replaced by arcs over $I$, all other boundary arcs unchanged, so that the map $B_{j}^{+}(0) \rightarrow B_{j}$ covers each point in $B \cap\{\Re W<0\} j$ times, and each point in $B \cap\{\Re W>0\} j-2$ times. The boundary correspondence $B_{j} \rightarrow B_{j}^{-}$remains the identity on all but these two arcs over $I$, while each of the two correspondences $S^{+} \rightarrow I$ rigidly compresses the arc-length element by the ratio $1: \pi$. Thus $B_{j}^{-}$is a simply-connected domain, a quadralateral, whose boundary is the union of the two arcs over $I$, one component projecting on $S^{+}$, with the remaining boundary component covering $S^{+}$a total of $j-2$ times and $S^{-} j$ times. Note that on a circuit of $\partial B_{j}^{-}$, the arc $I$ is traversed twice, each time with the same orientation. In this way we have described a $q c$ map $B_{j} \rightarrow B_{j}^{-}$, and it is straightforward to see that we may arrange dilatation independent of $j$.

The $\left\{B_{j}^{+}\right\}$, as noted above, are constructed in a parallel manner, except that two arcs over $S^{-}$will be replaced by arcs over $I$, and the branch point now lies over $W=p$. The dilatation of these maps is also uniformly bounded for $2 \leq j$, now with $I$ covered twice, each with orientation opposite to that from the $\left\{B_{j}^{-}\right\}$. Finally, we add one (univalent) cover $B^{*}$ which covers $B^{+}:=B \cap\{\Re W>0\}$. 
Now recall the $\operatorname{arcs} I_{k}^{ \pm}$introduced in $\S 4$, and note that relative to $\Sigma_{Z}^{\prime}$, $\partial^{-} \Sigma_{Z}$ has the larger argument. Using these $I_{k}^{ \pm}$, we divide $\Sigma_{Z}^{\prime}$ into one 'triangle' $Q_{0}$ and quadralaterals $Q_{k}, k \geq 1$. Thus $\partial Q_{0}$ will have as two sides the arcs $\left[Z_{0}, Z_{1}^{ \pm}\right] \subset \partial^{ \pm} \Sigma_{Z}$ as well as the segment $\left[Z_{1}^{-}, Z_{1}^{+}\right]$through $\Sigma_{Z}^{\prime}$. When $k \geq 1, \partial Q_{k}$ consists of $\operatorname{arcs} I_{k}^{-}, I_{k}^{+}$and the segments through $\Sigma_{Z}^{\prime}$ joining these endpoints. In view of (16), it is advantageous to take $\varepsilon(x)$ in (7) with

$$
\begin{aligned}
\varepsilon(x) & =\frac{2}{n(x)\left(1+h^{\prime}(x)^{2}\right)^{1 / 2}} \\
& =(1+o(1)) \frac{4 \pi}{\left(1+h^{\prime}(x)^{2}\right)^{1 / 2}} e^{-(1 / 2) \int_{0}^{x}\left(1+h^{\prime}(u)^{2}\right)^{1 / 2} d u} .
\end{aligned}
$$

The strip $\Sigma_{Z}^{\prime}=\bigcup_{k \geq 0} Q_{k}$ will be sent to $B=\{|W|<1\}$ with boundary values compatible with $H$ from $\S 3$, now to be made precise. Let $\sigma$ be the arc length on $I \cup S$ and $s$ be the arc length in the $Z$-plane. First, let $\psi_{0}: Q_{0} \rightarrow S \cap\{W<0\}$ with $d \sigma / d s$ constant on each segment $\left[Z_{0}, Z_{1}^{ \pm}\right]$and the segment $\left[Z_{1}^{+}, Z_{1}^{-}\right] \subset \Sigma_{Z}^{\prime}$, which corresponds under $\psi_{0}$ to $I$.

For the general case we have

Lemma 5. For $k \geq 1$ we may define $\Psi_{k}: Q_{k} \rightarrow B_{j}=B_{j}(k)$, with $2 \leq j(k) \leq$ $J\left(\rho^{*}\right)<\infty$, so that the arc-length correspondence is constant on each boundary segment, and

$$
\left\|\mu_{\Psi k}\right\|_{\infty}<\mu_{k}=\mu\left(\rho^{*}\right)<1 .
$$

Proof. Let us suppose that $I_{k}^{+} \cap S(\log x) \neq \varnothing$ for some $x \in J_{n}$. We have arranged that the image of $I_{k}^{-}$under $H$ cover $S^{+}$or $S^{-}$once, and that of $I_{k}^{+}$cover $S^{+} \cup S^{-}$ $q(k)$ times where $1 \leq q(k) \leq Q(n)<\infty$. Take $j(k)=1+q(k)$, and factor $\Psi=\Psi_{n}$ as $\Psi: Q_{k} \rightarrow \square \rightarrow B_{j}$ (here $\square$ is a square) again so that the arc-length correspondence is constant on each boundary arc. The dilatation of the map $\square \rightarrow B_{j}$ is readily controlled by $\rho^{*}$ : The two boundary segments of $B_{j}$ corresponding to $I$ are sent to opposite sides of $\square$, while the remaining sides of $\square$ correspond to covering $S^{ \pm}$ once and $(2 q(k)-3)$ times (this asymmetry due to the more rapid covering from $\left.\partial^{+} \Sigma_{Z}=\partial^{-} \Sigma_{Z}^{-}\right)$. The boundary of $Q_{k}$ consists of two line-segments through $\Sigma_{Z}^{\prime}$ and (due to (3) ) two near-radial segments on $\partial \Sigma_{Z}^{\prime}$, and (17) is made so that their side-lengths are comparable in a manner independent of $k$ (so long as they are made with $S(\log x) \cap Q_{k} \neq \varnothing$ with $\left.x \in J_{n}\right)$. Although these quadralaterals degenerate as $h^{\prime} \rightarrow \infty$, in the range $h^{\prime}<h\left(\rho^{*}\right)$ the mapping sending these arcs to the sides of $\square$ may be taken with dilatation uniformly bounded.

The function $H$ will be extended to $H^{*}$ in $\mathbb{C}$ as

$$
H^{*}(Z)= \begin{cases}H(Z) & \left(Z \in \Sigma_{Z}\right) \\ \Psi_{k}(Z) & \left(Z \in Q_{k} \subset \Sigma_{Z}^{\prime}\right),\end{cases}
$$

and $H^{*}$ is continuous in the plane.

We now make precise the data in (3) so that $H^{*}$ is transformed to the solution $f$ from (15). 


\section{Beltrami EQUATION}

The next lemma follows from normal families.

Lemma A. Corresponding to each $\eta>0, K<\infty$ are $M<\infty, \delta>0$ so that if $\psi$ is a homeomorphism of the plane fixing $z=0,1$ which is $K$-quasiconformal on $\left\{M^{-1}<|z|<M\right\}$ with

$$
\int_{S(r)}\left|\mu_{\psi}\left(r e^{i \theta}\right)\right| d \theta<\delta \quad\left(M^{-1}<r<M\right),
$$

where $\mu_{\psi}(z)=\left(\psi_{z}(z)+\psi_{\bar{z}}(z)\right) /\left(\psi_{z}(z)-\psi_{\bar{z}}(z)\right)$, then

$$
(1 \leq) \frac{\max _{\theta}\left|\psi\left(r e^{i \theta}\right)\right|}{\min _{\theta}\left|\psi\left(r e^{\theta}\right)\right|}<1+\eta \quad(1 / 2<r<2) .
$$

This lemma determines the intervals $J_{n}=\left[x_{n}, x_{n+1}\right]$ through which occur the stages of the construction implicit in (3) and (4). Choose the sequence $\left\{\eta_{n}\right\}$ in accord with Lemma 4 . Now consider a fixed $n \geq 0$, and since $h^{\prime}(t)<\rho_{n}^{*}$ when $Q_{k} \cap S(\log x) \neq \varnothing$ for $x \in J_{n}$, we have from Lemma 5 that $\left\|\mu_{\Psi_{k}}\right\|<\kappa_{n}<1$, or $\Psi_{k}$ is $K_{n}:=\left(1+\kappa_{n}\right) /\left(1-\kappa_{n}\right)$-quasiconformal. Lemma A produces sequences $\left\{M_{n}\right\}$ and $\left\{\delta_{n}\right\}$ which are now used.

To get a lower bound for each $x_{n}$, note from (16) and the second expression in (17): Given any $\varepsilon_{0}$, we may choose $x$ so large that independent of any data of $h(x)$, we have $\varepsilon(x)<\varepsilon_{0}$ if $x>x_{0}\left(\varepsilon_{0}\right)$. We thus take $x_{n}$ so that when $x>x_{n}-\log M_{n}$ we have

$$
\varepsilon(x)<\frac{\delta_{n}}{2 K_{n}} .
$$

This ensures that if $x>x_{n}-\log M_{n}$ and $S(\log x) \cap\left(Q_{k}\right) \neq \varnothing$,

$$
\int_{S\left(e^{x}\right) \cap \Sigma_{Z}^{\prime}}\left|\mu_{H^{*}}\left(r e^{i \theta}\right)\right| d \theta<\varepsilon_{0} \cdot K_{n}<(1 / 2) \delta_{n} .
$$

On the other hand, we see from the formula (10), Proposition 11, and (9) that

$$
\int_{S(r) \cap \Sigma_{Z}}\left|\mu_{H^{*}}\left(r e^{i \theta}\right)\right| d \theta
$$

is controlled by $\mu_{p}$ and $\mu_{\varphi}$, and consequently, when $\log r \in J_{n}$, by (3) and $\varepsilon(x)$. Thus when $x \in J_{n}, h^{\prime}(x)$ is bounded by (3) and $\mu_{\varphi}$ is controlled by $\eta=\eta_{n}$, and so we may increase $x_{n}$ if necessary to ensure that

$$
\int_{S(r)}\left|\mu_{H}\left(r e^{i \theta}\right)\right| d \theta<(1 / 2) \delta_{n} \quad\left(\log r>x_{n}-\log M_{n}\right) .
$$

This together with Lemma A implies that the homeomorphic normalized solution $\lambda(z)$ to the Beltrami equation $\lambda_{\bar{z}}=\mu_{H^{*}}(z) \lambda_{z}(z)$ is Hölder continuous with exponent $\tau$ (in fact this exponent may be taken as close to one as desired; we need only that it may be considered independent of $n$ or the choice of $\rho$ in Theorem 1). Thus

$$
\log |\zeta(Z)|=(1+o(1)) \log |Z| \quad(|Z| \rightarrow \infty),
$$

so that the entire function $f$ in (18) has the same order and lower order as $H^{*}$. Since all solutions to $|f(\zeta)|=1$ are related to those of $H^{*}(\zeta) \mid=1$ by $\lambda$, Lemma 3 shows that $f$ has the desired growth. We have constructed $H$ so all singularies are over $\pm p, \infty$, and thus (15) gives us that $f \in S_{3}$. 


\section{REFERENCES}

[1] I. N. BAKer, J. Kotus, Y Lü, Iterates of meromorphic functions. IV. Critically finite functions, Results Math 22 (1992), 651-656. MR1189754 (94c:58166)

[2] W. Bergweiler, A. Eremenko, On the singularities of the inverse to a meromorphic function of finite order, Rev. Mat. Iberoamericana 11 (1995), 355-373. MR1344897 (96h:30055)

[3] D. Drasin, A. Weitsman, Meromorphic functions with large sums of deficiencies, Advances in Math 15 (1975), 93-126. MR0355051(50:7528)

[4] A. Eremenko, M. Yu. Lyubich, Dynamical properties of some classes of entire functions, Ann. Inst. Fourier (Grenoble) 42 (1992), 989-1020. MR1196102 (93k:30034)

[5] H. KÜNZI, H. WitTich, The distribution of a-points of certain meromorphic functions, Mich. Math. J. 6 (1959), 105-121. MR0104808(21:3561)

[6] S. Merenkov, Rapidly growing entire functions with three singular values, preprint.

[7] O. Teichmüller, Eine Umkehrung des zweiten Hauptsatzes der Wertverteilungslehre, Deutsche Mathematik 2 (1937), 96-107.

[8] H. Wiтtich, Neuere Untersuchungen über eindeutige analytische Funktionen (German) (Ergebnisse der Mathematik und ihrer Grenzgebite (N. F.) Heft 8), Springer Verlag, 1955. MR0077620 (17:1067a)

Department of Mathematics, Purdue University, West Lafayette, Indiana 47907-2067

E-mail address: drasin@math.purdue.edu 